\title{
A Note on Expanding Networks and Monopoly Pricing
}

J.J. Gabszewicz and F. Garcia

Discussion Paper 2005-63

\section{Département des Sciences Économiques de l'Université catholique de Louvain}




\title{
A Note on Expanding Networks and Monopoly Pricing
}

\author{
Jean J. Gabszewicz*and Filomena Garcia ${ }^{\dagger}$
}

CORE Discussion Paper 2005/95

\begin{abstract}
We obtain explicitly the optimal path of prices for a monopolist operating in a network industry during a finite number of periods. We describe this optimal path as a function of network intensity and horizon length and show that the prices are increasing in time and that, for very low network intensity, or very high horizon length, the monopolist will offer the good at zero price in the initial period.
\end{abstract}

${ }^{*}$ CORE-UCL

${ }^{\dagger}$ CORE-UCL. Filomena Garcia gratefully acknowledges the financial support from Fundaçao Gulbenkian and CORE. The usual disclaimer applies. 


\section{Introduction}

In this paper we analyze the pricing decision of a monopolist who sells a good subject to network externalities for a finite number of periods. We consider that the network takes one period of time to be constituted so that consumers derive utility in each period from the volume of past sales (see Rohlfs (1974), Katz and Shapiro $(1985,1994)$ and Farrell and Saloner (1985)). This type of network externalities can be found when the quality of the good depends on some "word of mouth" or "learning by doing" process, according to the terminology in Bensaid and Lesne (1996). Early consumers allow firms to improve their product, or to offer better services and assistance in later periods, which increases the benefit of consuming the good for later users. Our analysis is, in particular, appropriate for reputation goods (see Rogerson (1982)), or goods which require a learning period. For instance, consider the market for a reputation good when reputation increases with the size of its market. The initial buyers of such a good spread information about how to use it and enjoy its consumption. The group of consumers who buy the good initially can be viewed as a network, improving the utility of its potential consumers and thereby the potential profits opportunities of the firm. This is the case of several products resulting from recent innovation, and whose characteristics can only be revealed by direct consumption. Also for many goods, the size of the initial network of buyers determines the availability of complementary services the seller would be willing to develop around the variant which is initially supplied. Examples are: a bank and the number of branches which increases with the number of its customers, a new machine and the number of its components when they are available to subsequent buyers, like in the case of a computer whose number of compatible programs increases with the number of its initial buyers. Notice however that such product improvements or reputation effects need some period of time to be finalized, creating a time lag between the creation of the network, and its effect on the utility of subsequent buyers. This structure of preferences implies that consumers always consider past consumers' decisions before decid- 
ing to acquire the good or not. Consequently, the monopolist's pricing decision must consist in an intertemporal strategy: the price chosen in early periods influences future sales and profits.

In this note we uncover the monopolist's optimal strategy and study how it depends on the intensity of the preference for the network and on the horizon's length. In initial periods, the network effect is mild and thus the surplus that the monopolist could extract from the consumers by setting the instantaneous monopoly price is low. On the contrary, setting a lower price attracts consumers and increases the demand in future periods. The incentive to set low prices is, nevertheless decreasing in time because the closer the horizon, the weaker the interest of increasing further future demand. As a consequence, we first observe that the path of optimal prices is increasing through time. We also observe that this path of prices approaches the sequence of instantaneous monopoly prices corresponding to the sequence of accumulated demands, while being exactly equal to this monopoly price in the last period.

Furthermore,for large values of network intensity, we show that the monopolist chooses initial prices to be zero, indicating that he would even set a negative price if this would be admissible. We also show that, in this context, the monopolist selects a path of prices which increases through time, and that this path is steeper than when the network intensity is weaker.

The initial zero price phenomenon is frequently observed. Banks usually offer low rates for new users, software is usually offered at very low price (or, even, given for free) in initial periods (whereas updates are expansive); similarly, phone companies or network providers have low price deals for new adherents (See Ackere and Reyniers (1995)). In this note, we provide a necessary and sufficient condition under which the initial price is zero, implying that the seller has interest in offering free sample of the good in the first period. To the best of our knowledge, this condition has not been discussed so far in the literature.

Our note relates to previous literature on the pricing of durable goods and experience goods. In particular, Cabral et al. (1999) study monopoly pricing in a network industry and conclude that introductory pricing occurs if consumers 
are price takers, if there is incomplete information about demand or asymmetric information about cost. We show that introductory pricing occurs in a setup where information is complete and symmetric. Clarke et al. (1982) discuss, within a continuous time framework, the consequences on monopoly pricing of experience effects in demand. They are also led to the conclusion that the path of prices should be increasing through time. Dhebar and Oren $(1985,1986)$, analyzing linear and nonlinear pricing strategies for the monopolist, also conclude that his profit margins should be increasing. Our note contributes to this discussion by providing explicitly the optimal path of prices. We also obtain comparative statics results related to the length of the horizon and to the intensity of the network.

\section{The model and results}

Consider a profit maximizing monopolist that sells a durable good at constant average cost set to zero. The monopolist will be active in the market for $T$ periods.

At each period $t, t=1, \ldots, T$,a new cohort of consumers enters the market. Consumers value the good for its intrinsic utility and for the network benefits that it entails. The more consumers buy the good the higher is its value for each individual. Consumers are differentiated according to their stand alone value. They are distributed uniformly in $[0,1]$ by increasing order of intrinsic valuation for the good.

The demand for the good at time $t$, is denoted by $D_{t}\left(p_{t}, p_{t-1}, \ldots, p_{1}\right)$. The utility of agent $v$ at time $t$ is given by:

$$
u_{t}\left(v ; p_{t-1}, \ldots, p_{1}\right)=v+\alpha \sum_{j=1}^{t} D_{t-j}\left(p_{t-j}, \ldots, p_{1}\right) ;
$$

The parameter $\alpha, \alpha>0$, measures the intensity of the network effect.

First, we derive the demand function for the product in each period $t, t=$ $1, \ldots, T$. Since the consumer, in her decision problem at period $t$, only considers 
the network constituted in the past periods, the functional form of demand in each period is independent of the subsequent periods and depends only on prices $p_{\tau}, \tau=1, \ldots . t$. Let us start with $T=2$. The demand at period 1 is given by:

$$
D_{1}\left(p_{1}\right)=1-p_{1}, 1 \geq p_{1} \geq 0 .
$$

In period 2, taking into account the network effect created in period 1 , we identify the consumer $\widehat{v}$ who is indifferent between buying the product and not buying in period 2 by the condition

$$
v+\alpha\left(1-p_{1}\right)-p_{2}=0
$$

namely, $\widehat{v}=p_{2}-\alpha\left(1-p_{1}\right)$. Thus, demand in period 2 is given by:

$$
D_{2}\left(p_{2}, p_{1}\right)=1-p_{2}+\alpha\left(1-p_{1}\right)
$$

Likewise, in period 3 and taking into account the network effect in periods 1 and 2, the consumer who is indifferent between buying the product and not buying in period 3 , denote it by $\widehat{\widehat{v}}$, is given by the condition

$$
v+\alpha\left(1-p_{1}+1-p_{2}+\alpha\left(1-p_{1}\right)\right)-p_{3}=0,
$$

namely, $\widehat{\widehat{v}}=p_{3}-\alpha\left(1-p_{1}+1-p_{2}+\alpha\left(1-p_{1}\right)\right)$. Thus, the demand in period 3 obtains as

$$
D_{3}\left(p_{3}, p_{2}, p_{1}\right)=1-p_{3}+\alpha\left(1-p_{1}+1-p_{2}+\alpha\left(1-p_{1}\right)\right) .
$$

Proceeding by induction, it is easy to see that the demand in period $t \leq T$ obtains as

$$
D_{t}\left(p_{t}, \ldots, p_{1}\right)=(1+\alpha)^{t-1}-\alpha \sum_{j=2}^{t}(1+\alpha)^{t-j} p_{j-1}-p_{t} .
$$

Notice that, when $\alpha>1$, the demand might react with more intensity to previous prices, $p_{2}$ and $p_{1}$, than to current price. This is so because, under this condition, lower initial prices increase the value of the good for subsequent users. 
On the contrary, for $\alpha<1$, the effect of initially low prices tends to vanish with time. An alternative expression for the demand obtains as

$$
D_{t}\left(p_{t}, \ldots, p_{1}\right)=1-p_{t}+\alpha \sum_{j=1}^{t} D_{t-j} .
$$

The demand in period $t$ is a linear decreasing function of $p_{t}$ with the constant term increasing in $t$. All the above expressions for demands at period $t$ must of course satisfy the fact that demand can never exceed 1.

The total profit function with horizon $T$ writes as:

$$
\begin{aligned}
\Pi\left(T ; p_{T}, \ldots, p_{1}\right) & =\sum_{k=1}^{T} p_{k} D_{k} \\
& =\sum_{k=1}^{T} p_{k}\left((1+\alpha)^{k-1}-\alpha \sum_{j=2}^{k}(1+\alpha)^{k-j} p_{j-1}-p_{k}\right) .
\end{aligned}
$$

\section{3 "Weak" network intensity}

Consider first that the condition

$$
\alpha<\frac{1}{T-1}
$$

is satisfied by the parameters of the model.

Proposition 1 Under C.1, the set of values given by

$$
p_{t}(T)=\frac{1-(T-t) \alpha}{2-(T-1) \alpha}, t=1, \ldots, T
$$

constitutes the unique solution to the monopolist's profit maximization problem.

Proof. We differentiate $\Pi\left(T ; p_{T}, \ldots, p_{1}\right)$ with respect to $p_{1}, \ldots, p_{T}$ and, by the first order necessary conditions for an interior maximum, we get a system of $T$ equations defined by

$$
\frac{\partial \Pi\left(T ; p_{T}, \ldots, p_{1}\right)}{\partial p_{s}}=0, s=1, \ldots, T
$$


or, equivalently

$(1+\alpha)^{s-1}-\alpha \sum_{j=2}^{s}(1+\alpha)^{s-j} p_{j-1}-2 p_{s}-\alpha \sum_{i=1}^{T-s} p_{s+i}(1+\alpha)^{i-1}=0, s=1, \ldots T$.

It is easy to see that, the vector of prices (4) is the unique solution to the above system whenever condition C.1 holds (notice that, $p_{t}(T)$ increases with $t$; accordingly, $p_{1}(T)=\frac{1-(T-1) \alpha}{2-(T-1) \alpha}$ is positive if, and only if, C.1 is satisfied). Now, it remains to check when the first order necessary conditions identified above are also sufficient for (4) being a solution to the maximization problem of the monopolist. The Hessian matrix associated with the maximization problem writes as

$$
A(T)=-\left[\begin{array}{ccccc}
2 & \alpha & \alpha(1+\alpha) & \ldots & \alpha(\alpha+1)^{T-2} \\
\alpha & 2 & \alpha & \ldots & \alpha(\alpha+1)^{T-3} \\
\alpha(1+\alpha) & \alpha & 2 & \ldots & \alpha(\alpha+1)^{T-4} \\
\ldots & \ldots & \ldots & 2 & \alpha \\
\alpha(\alpha+1)^{T-2} & \alpha(\alpha+1)^{T-3} & \alpha(\alpha+1)^{T-4} & \alpha & 2
\end{array}\right]
$$

The total profit function $\Pi\left(T ; p_{T}, \ldots, p_{1}\right)$ is strictly concave iff,

$$
\begin{aligned}
\operatorname{det}(A(T))= & (-1)^{T}((T-1) \alpha-2)(\alpha+2)^{T-1}<0, T \text { even } \\
& \text { or, } \\
\operatorname{det}(A(T))= & (-1)^{T}((T-1) \alpha-2)(\alpha+2)^{T-1}>0, T \text { odd }
\end{aligned}
$$

or, equivalently

$$
\alpha<\frac{2}{T-1} .
$$

This condition is trivially satisfied when $\alpha$ satisfies C. 1 and it also guarantees the uniqueness of the solution to the maximization problem.

To illustrate the above, the following table represents the sequence of optimal prices corresponding to various values of the horizon $T$. 


\begin{tabular}{|c|c|c|c|c|c|}
\hline$T=2$ & $\frac{1-\alpha}{2-\alpha}$ & $\frac{1}{2-\alpha}$ & & & \\
\hline$T=3$ & $\frac{1-2 \alpha}{2-2 \alpha}$ & $\frac{1-\alpha}{2-2 \alpha}$ & $\frac{1}{2-2 \alpha}$ & & \\
\hline$T=4$ & $\frac{1-3 \alpha}{2-3 \alpha}$ & $\frac{1-2 \alpha}{2-3 \alpha}$ & $\frac{1-\alpha}{2-3 \alpha}$ & $\frac{1}{2-3 \alpha}$ & \\
\hline$\ldots$ & $\ldots$ & $\ldots$ & $\ldots$ & $\ldots$ & \\
\hline$T$ & $\frac{1-(T-1) \alpha}{2-(T-1) \alpha}$ & $\frac{1-(T-2) \alpha}{2-(T-1) \alpha}$ & $\frac{1-(T-3) \alpha}{2-(T-1) \alpha}$ & $\ldots$ & $\frac{1}{2-(T-1) \alpha}$ \\
\hline
\end{tabular}

It is interesting to discuss briefly how this solution depends on the main ingredients of the model, namely, the time horizon $T$ and the network intensity parameter $\alpha$. In order to perform the desired comparative statics we easily compute the value of demand $D_{t}, t=1, \ldots, T$, at optimal prices $p_{t}(T), \ldots, p_{1}(T)$, namely

$$
D_{t}\left(p_{t}(T), \ldots, p_{1}(T)\right)=\frac{1}{2-(T-1) \alpha} .
$$

First notice that the value of each demand at period $t$ evaluated at optimal prices is smaller than 1. Furthermore, we find that this value is constant, given the horizon $T$. Finally, it is increasing with the horizon $T$ and decreasing with $\alpha$.

The period $t$-profits at the optimal solution $\pi_{t}(T)$ obtain as

$$
\begin{aligned}
\pi_{t}(T) & =p_{t}(T) \cdot D_{t}\left(p_{t}(T), \ldots, p_{1}(T)\right) \\
& =\frac{1-(T-t) \alpha}{(2-(T-1) \alpha)^{2}}
\end{aligned}
$$

Therefore, the per-period profits $\pi_{t}(T)$ are increasing over time and also increasing with the network intensity $\alpha$ (under C.1).

Total profits evaluated at optimal prices, given the horizon $T$, obtain as

$$
\Pi\left(T, p_{t}(T), \ldots, p_{1}(T)\right)=\sum_{t=1}^{T} \pi_{t}(T)=\frac{T}{2(2-(T-1) \alpha)} .
$$

An immediate conclusion is that total profits are an increasing function of the horizon, as well as of the network intensity $\alpha$. 


\section{4 "Strong" network intensity}

By "strong network intensity", we mean that condition C.1 is violated. First, let us assume that

$$
\frac{1}{T-1} \leq \alpha \leq \frac{2}{T-1}
$$

As shown above, total profits are also strictly concave in this domain, but some, or all, components of the vector $\left\{p_{t}(T)=\frac{1-(T-t) \alpha}{2-(T-1) \alpha}\right\}_{t=1}^{T}$ must be equal to 0: all prices are to be nonnegative. When $\alpha$ increases beyond the value $\frac{1}{T-1}$, the first component of this vector to become smaller or equal to 0 at $(4)$ is $p_{1}(T)$. Then, how to characterize the optimal path of prices for $p_{2}(T), p_{3}(T), \ldots, p_{T}(T)$ ?

Proposition 2 Under C.3, the set of values given by

$$
\begin{aligned}
& p_{1}(T)=0 \\
& p_{t}(T)=(t-1) \alpha, t=2, \ldots, T
\end{aligned}
$$

constitutes the unique solution to the monopolist's profit maximization problem.

Proof. First, notice that the domain of values for which $p_{1}(T)=\frac{1-(T-1) \alpha}{2-(T-1) \alpha} \leq 0$, namely, $\left\{\alpha: \frac{1}{T-1}<\alpha<\frac{2}{T-1}\right\}$, strictly includes the domain for which $p_{1}(T+$ $1) \leq 0$. Consequently, the strongest condition for having $p_{1}(T)=0$ obtains for the horizon $T=2$ so that, whatever the value of the horizon $T \geq 2$, we must have $p_{1}(T)=0$. We shall now prove the proposition by induction. Consider first $T=2$. Then, the second and third period demands are equal to:

$$
\begin{aligned}
D_{2}\left(p_{2}, p_{1}\right) & =1-p_{2}+\alpha \\
D_{3}\left(p_{3,}, p_{2}, p_{1}\right) & =1-p_{3}+\alpha\left(1-p_{2}+\alpha\right) .
\end{aligned}
$$

We show that, under condition C.3, the optimal second period price $p_{2}(2)$ is equal to $\alpha$. Total profit $\Pi\left(2, p_{2}, 0\right)$ is then equal to $1+p_{2}\left(1-p_{2}+\alpha\right)$. The first-order condition writes as

$$
1-2 p_{2}+\alpha=0
$$


which is satisfied for $p_{2}=\frac{1+\alpha}{2}$. Substituting this value in $D_{2}\left(p_{2}, p_{1}\right)$, we see that $D_{2}=\frac{1+\alpha}{2}$, which exceeds 1 , given that, by condition C.3, we have $\alpha>1$. Accordingly, this value should be discarded as a solution to the maximization problem, and the optimal solution obtains at the highest value for $p_{2}$ keeping the demand smaller than 1 , namely, $p_{2}=p_{2}(2)=\alpha$.

Finally we must show that, if the path of prices $(0, \alpha, 2 \alpha, \ldots(T-2) \alpha)$ is optimal up to $T-1$, then the optimal price path up to $T$ is given by the sequence $(0, \alpha, 2 \alpha, \ldots(T-1) \alpha)$. So, assume that $(0, \alpha, 2 \alpha, \ldots(T-2) \alpha)$ is the optimal sequence of prices when the horizon is $T-1$. The demand function $D_{T}$ then writes as $1-p_{T}+\alpha(T-1)$, with corresponding total profits equal to $p_{T}\left(1-p_{T}+\alpha(T-1)\right)$. The first order condition writes as

$$
1-2 p_{T}+\alpha(T-1)=0
$$

which is satisfied for $p_{T}=\frac{1+\alpha(T-1)}{2}$. Substituting this value in $D_{T}$, we see that, by condition C.3 $\left(\alpha \geq \frac{1}{T-1}\right)$, the corresponding demand exceeds the value 1 , which is not admissible. Then, $p_{T}(T)=\alpha(T-1)$, which is the price that corresponds to demand $D_{T}=1$.

Now, consider the case in which condition

$$
\alpha>\frac{2}{T-1}
$$

holds. Then, the total profit function is no longer concave, but convex. Accordingly, the solution to the monopolist's problem occurs at the boundary of the admissible domain of prices, namely $\left\{\left(p_{1}, \ldots, p_{T}\right): 0 \leq D_{t}\left(p_{t}, \ldots, p_{1}\right) \leq 1, t=1, \ldots, T\right\}$. In what follows we show:

Proposition 3 Under C.4, the set of values given by

$$
\begin{aligned}
& p_{1}(T)=0 \\
& p_{t}(T)=(t-1) \alpha, t=2, \ldots, T
\end{aligned}
$$

constitutes the unique solution to the monopolist's profit maximization problem. 
Proof. Suppose first that $T=2$ and that C. 4 is satisfied, namely, $\alpha \geq \frac{1}{2}$. Then, the total profit function of the monopolist writes as:

$$
\Pi\left(2, p_{2}, p_{1}\right)=p_{1}\left(1-p_{1}\right)+p_{2}\left(1-p_{2}+\alpha\left(1-p_{1}\right)\right)
$$

which is a convex function in $p_{1}$ and $p_{2}$. The optimal solution must lie on the boundary, namely, $p_{1}=0$ and $p_{2}=\alpha$, the prices for which both demands are equal to 1 . Then proceeding as in the proof of Proposition 2, it is easy to show that the sequence of prices $0, \alpha, \ldots,(T-1) \alpha$ constitutes the optimal monopoly solution when C.4 holds.

\section{Conclusion}

Our analysis is developed along the values of two major parameters, namely, the network intensity and the horizon length, which are related to each other by conditions C.1 to C.4. When the network intensity and the horizon length meet condition C.1, the market is never saturated: given the weakness of network intensity, the horizon is not sufficiently far for the monopolist to cover the market and keep it covered for the remaining periods. When conditions C.2 and C.3 are fulfilled, namely, when network intensity is sufficiently strong, the monopolist is induced to saturate the market from the very beginning, and keep it covered for all subsequent periods. Notice that when the horizon length tends to infinity, the domain of network intensity values to which corresponds an interior solution, i.e. in which zero pricing is not practised, tends to a set of measure zero. Thus we conclude that, when the horizon is sufficiently remote, zero pricing in the starting period should be the rule.

The vector of prices identified in the optimal policies above corresponds to a solution in which the monopolist is not allowed to revise his strategy. However, we have checked that the solution would not be altered if this revision would be allowed. This property follows from the fact that consumers arrive in the market by successive waves and cannot delay consumption, which implies that they cannot arbitrate between periods when making their purchase decisions. 


\section{References}

[1] Ackere, A. and D. Reyniers, 1995, "Trade-ins and introductory offers in a monopoly", RAND Journal of Economics, 26 (1), 1995, 58-74

[2] Bensaid, B. and J.-P. Lesne, 1996, "Dynamic Monopoly Pricing with Network Externalities", International Journal of Industrial Organization, 14, $837-855$

[3] Cabral, L. , D. Salant and G. Woroch, 1999, "Monopoly Pricing with Network Externalities", International Journal of Industrial Organization, 17, $199-214$

[4] Clarke, F. and Darrough, M. and J. Heineke, 1982, "Optimal pricing policy in the presence of experience effects", The Journal of Business, 55(4), 517530

[5] Dhebar, A. and S. Oren, 1985, "Dynamic nonlinear pricing in networks with interdependent demand", Operations Research, 34 (3), 384-394

[6] Dhebar, A. and S. Oren, 1985, "Optimal dynamic pricing for expanding networks", Marketing Science, 4 (4), 336-351

[7] Farrell, J. and G. Saloner, 1985, "Standardization, compatibility,and innovation", Rand J. Econom, 16, 70-83.

[8] Katz, M. and C. Shapiro, 1985, "Network effect, competition, and compatibility", Amer. Econom. Rev., 75(3), 424-440

[9] Katz, M. and C. Shapiro, 1994, "System competition and network effects", J. Econom. Perspectives, 8(2), 93-115

[10] Rogerson, W., 1983, "Reputation and product quality", The Bell Journal of Economics, 14 (2), 508-516

[11] Rohlfs, J. (1974), "A theory of interdependent demand for a communication service", Bell Journal of Economics, 5, 16-37 
Département des Sciences Économiques de l'Université catholique de Louvain

Institut de Recherches Économiques et Sociales

Place Montesquieu, 3

1348 Louvain-la-Neuve, Belgique 